\title{
Féeries
}

Études sur le conte merveilleux, XVII $-\mathrm{XIX}{ }^{\mathrm{e}}$ siècle

\section{Vues de l'esprit : la question de la sensibilité dans les fictions merveilleuses de Tiphaigne de La Roche}

Figments of the Imagination: the Question of Sensibility in Tiphaigne de La Roche's Marvelous Fictions

\section{Guilhem Armand}

\section{OpenEdition}

Journals

\section{Édition électronique}

URL : http://journals.openedition.org/feeries/1671

DOI : 10.4000/feeries. 1671

ISSN : 1957-7753

\section{Éditeur}

UGA Éditions/Université Grenoble Alpes

Édition imprimée

ISBN : 978-2-37747-075-4

ISSN : 1766-2842

\section{Référence électronique}

Guilhem Armand, « Vues de l'esprit : la question de la sensibilité dans les fictions merveilleuses de Tiphaigne de La Roche », Féeries [En ligne], 15 | 2018, mis en ligne le 14 février 2019, consulté le 08 septembre 2020. URL : http://journals.openedition.org/feeries/1671 ; DOI : https://doi.org/10.4000/ feeries. 1671

Ce document a été généré automatiquement le 8 septembre 2020.

(c) Féeries 


\title{
Vues de l'esprit : la question de la sensibilité dans les fictions merveilleuses de Tiphaigne de La Roche
}

\author{
Figments of the Imagination: the Question of Sensibility in Tiphaigne de La \\ Roche's Marvelous Fictions
}

\section{Guilhem Armand}

Médecin de formation, Charles-François Tiphaigne de La Roche a consacré sa thèse, puis son premier ouvrage publié, à la question de la sympathie. Comme l'a montré Philippe Vincent ${ }^{1}$, c'est à partir de cette théorie de la sympathie que Tiphaigne explique le fonctionnement des sens et des sensations, ainsi que des sentiments (comme l'amour). En 1747, dans sa thèse de médecine, Pour savoir si l'explication de la sympathie et de l'antipathie doit être cherchée dans la matière transpirante, il s'intéresse à la "matière transpirante ", une matière invisible qui s'exhale continuellement des corps et qui est à l'origine de toutes les sensations. Ce sensitivisme vitaliste s'appuie sur une autre théorie, celle des esprits animaux, c'est-à-dire, pour reprendre la définition de Descartes, "des corps très petits et qui se meuvent très vite " "produits dans le cerveau » : "[...] à mesure qu'il en entre quelques-uns dans les cavités du cerveau, il en sort aussi quelques autres par les pores qui sont en sa substance, lesquels pores les conduisent dans les nerfs, et de là dans les muscles, au moyen de quoi ils meuvent le corps en toutes les diverses façons qu'il peut être mû́."»

1 Issue de Galien, cette théorie connaît un renouveau à la suite des travaux de Descartes, puis de nombreux anatomistes et philosophes tout au long du XVIII $^{\mathrm{e}}$ siècle $^{3}$. Ressortissant aux deux domaines, à l'instar de celle de la sympathie ${ }^{4}$, elle a entraîné un grand nombre de variations. Et Tiphaigne semble s'être servi de ce flou conceptuel à des fins poétiques. Dès L'Amour dévoilé ou le Système des sympathistes, en 1749, il opère un premier glissement en parlant non plus de "matière transpirante », mais de " matière sympathique ». Le raccourci permet ainsi de préparer un autre glissement, celui des 
esprits animaux de la médecine de l'époque, que l'on ne voit pas mais qui expliquent l'insuffisance de nos sens, aux esprits élémentaires que les contes donnent à voir et qui suggèrent la toute-puissance de l'imagination.

C'est ce paradoxe d'une philosophie à la fois empiriste, dans la lignée de Locke, et spiritualiste - explicitement contre les lectures de Locke par ses contemporains - qu'il s'agit d'explorer, afin de tenter de démontrer qu'il innerve la poétique des étranges et inclassables œuvres de Tiphaigne de La Roche, notamment celle de ses fictions merveilleuses.

\section{De l'insuffisance des sens et des limites de la connaissance}

La pensée de Tiphaigne de La Roche est assez complexe en ce qu'elle relève d'un mélange fort personnel de plusieurs épistémologies, allant des sciences occultes à la physiologie moderne, s'inspirant des théories alchimiques comme des découvertes les plus récentes. Le subtil mélange entre son empirisme scientifique et sa réévaluation des songes perçus comme moteurs de la connaissance a pu être interprété comme un élément rapprochant Tiphaigne de Diderot, par exemple. Ainsi, Florence Boulerie opère un parallèle entre les styles des deux auteurs ${ }^{5}$. Ce rapprochement a pu être fait dès la parution des Bigarrures philosophiques, attribuées à Diderot par une partie du public, d'où ce démenti sévère de Grimm :

Un médecin de Caen, nommé Tiphaigne, a publié deux petits volumes assez bien imprimés sous le titre de Bigarrures philosophiques. Ce sont assurément des bigarrures, mais elles ne paraîtront pas philosophiques. Il y a cependant eu des gens d'assez peu de goût pour attribuer cet ouvrage à M. Diderot. C'est ne savoir point distinguer la vraie philosophie avec ce qui n'en a qu'un faux air6.

Mais la pensée de Tiphaigne rejoint bien celle des Lumières sur un point : son antisystémisme qui repose sur la certitude des limites de la connaissance rationnelle des choses, en raison de l'insuffisance des sens humains. Ce point constitue à la fois un leitmotiv de ses œuvres et un moteur de ses voyages imaginaires et de ses songes satiriques. Ainsi, dans le Voyage aux Limbes (au début du second volume des Bigarrures philosophiques ${ }^{7}$ ), l'auteur met en scène son héros-voyageur dans un périple imaginaire le conduisant dans cette région méconnue où séjournent les philosophes morts. Ces Champs-Élysées resitués dans l'espace interstellaire rappellent au lecteur la « Province des Philosophes » où se dirigeait Dyrcona, plein d'espoir de réponses ${ }^{8}$, à la fin des États et Empires du Soleil, mais aussi les « espaces imaginaires » cartésiens parodiés par le Père Daniel dans le Voyage du Monde de Descartes à la fin du siècle précédent ${ }^{9}$. Et c'est davantage dans cette lignée que se situe Tiphaigne qui met en scène des savants, ainsi que des factions philosophiques réunies tantôt en écoles, tantôt en spécialités (physique, métaphysique), qui ne s'entendent pas du tout entre eux. L'échec d'un savoir fondé sur la science moderne qui allie empirisme et rationalisme se manifeste non seulement dans les disputes sans fin qui animent les savants habitants des Limbes, mais aussi dans le constat du voyageur parvenu aux «Plaines de la physique » :

Tous les jours on y découvre des nouveautés, et jamais on ne découvrira tout. Le malheur est qu'on ne peut rien approfondir. On y voit beaucoup de choses, mais on les voit mal, quoiqu'on ne manque ni de lunettes ni de microscopes. Les objets qui intéressent le plus la curiosité sont ou trop petits, ou trop grands, les yeux s'y perdent. Ce qu'on croit apercevoir, on ne fait souvent que se le figurer : de là vient que 
vis-à-vis du même objet, l'un voit une chose, l'autre une autre, et souvent aucun ne voit ce qui en est. C'est un pays d'illusions \& l'on ne peut trop y être sur ses gardes ${ }^{10}$. - « qui apparaît à la vue »-, est réduite à un champ d'illusions. L'isotopie de la vue parcourt l'œuvre de Tiphaigne de La Roche, mais sur le mode de la négation pour dénoncer, du début à la fin, les limites du postulat empiriste voir = savoir, et aboutir à une réécriture pessimiste du constat socratique :

Car enfin, ce qu'on voit n'est rien, comparé à ce qu'on ne voit pas. Il n'y a qu'un ignorant qui puisse s'applaudir de ses connaissances, le savant demeure épouvanté à la vue de ce qu'il ne sait pas ${ }^{11}$. Je sais que je ne sais rien, semble répéter Tiphaigne. Dans Giphantie (1760), l'auteur revient malicieusement sur ces « nouveautés » découvertes «tous les jours »; ce sont les effets des amusements d'Esprits élémentaires se jouant des humains, comme l'explique le Préfet au bien nommé chapitre « Contre-sens » :

Un naturaliste s'étonne quelquefois de trouver des corps naturels, qu'aucun autre savant, avant lui n'avait remarqués : c'est que nous en avons pourvu la terre depuis peu, et c'est ce qu'il n'a garde de soupçonner.

Telle espèce de plante subsiste encore, mais languit depuis plusieurs siècles, perd ses qualités et trompe le médecin, qui tous les jours manque son objet. On accuse l'art; on ne sait pas que c'est la faute de la nature ${ }^{12}$.

Et tout ce conte merveilleux de Giphantie - de même que La Zazirocratie, publié en 1761 - est fondé sur une dialectique entre ce qu'on voit ou ne voit pas. Cependant, le docteur Tiphaigne ne cesse de se passionner pour les diverses découvertes de son temps, notamment en médecine, en témoigne le travail de reprises avec ajouts conséquents qui a présidé aux trois éditions d'Amilec ou la Graine d'homme ${ }^{13}$ (1753-1754), conte consacré à la question de la génération. Et l'on a montré ailleurs que les dissonances apparentes entre les théories exposées dans l'ouvrage par les Génies de la reproduction restituent en fait l'évolution des thèses de Maupertuis jusqu'à en opérer une habile et novatrice synthèse ${ }^{14}$. L'incipit d'Amilec présente le héros-narrateur en lecteur d'ouvrages savants sur la question de la génération, mais en proie au désespoir de l'ignorance :

Il y avait sept heures que j'étais enfermé dans mon cabinet. J’y étais opiniâtrement collé sur un volume assez ample, où il est parlé de la génération. Je le parcourus avec toute l'avidité dont est capable un homme qui ne sait rien, et qui brûle d'apprendre.

Que me resta-t-il de cette étude? ce qui reste de toutes celles de ce genre; des doutes. Rebuté de ce pénible exercice, aussi ignorant que je l'étais auparavant, je jetai là le volume, et me voilà à invectiver contre tout ce qui s'appelle physicien, naturaliste, médecin, philosophe, etc. ${ }^{15}$

Le héros s'endort et est visité en songe par un «jeune homme » qui s'avère être «le génie qui préside à la multiplication de l'espèce humaine » (p. 96) et qui va lui apporter les réponses tant désirées. Dans le laboratoire d'Amilec, il va voir ce qu'un microscope ne pouvait lui permettre de découvrir. Autrement dit, l'imaginaire onirique vient au secours des insuffisances de la science et de la perception humaine. Cependant, le merveilleux, chez Tiphaigne, n'agit pas simplement comme un palliatif. Si, comme dans d'autres fictions à vocation scientifique, la fiction prolonge allégoriquement l'effet du microscope, elle donne à voir non pas nécessairement plus précisément, mais autrement. Une étrange équivalence se pose ainsi entre savoir et rêver, théorisée au début des Bigarrures, dans les Visions d'Ibraïm: "Presque tout le monde dort 
continuellement, ce qu'on appelle être éveillé, n'est que dormir un peu moins. Deux espèces de gens veillent, peut-être, réellement, les hommes de génie, et les fous ${ }^{16}$." Ainsi, entre la vision fantaisiste d'un fou - ou d'un conteur ? - et le système d'un philosophe, la frontière devient aussi floue qu'entre la veille et le songe :

[...] quand nous rêvons, nous imaginons mille choses qui ne ressemblent à rien, nous faisons des systèmes universels, nous entreprenons de tout expliquer; et, plutôt que de rester court, nous donnons de l'intelligence à la poussière, et du raisonnement aux atomes ${ }^{17}$.

Le constat anatomique de l'insuffisance des sens fonde le postulat épistémologique des limites de la science humaine et donc une poétique de l'imaginaire onirique qui ouvre «la barrière » des champs du savoir ${ }^{18}$. L'ironique parti pris qui dévalue les systèmes pour rehausser le rêve comme voie d'accès à la connaissance, dès l'épître « Au Savants » d'Amilec ${ }^{19}$, devient aussi un leitmotiv des contes tiphaigniens ${ }^{20}$. Chez ce médecin - mais y croit-il ? - le songe n'est pas seulement un artifice littéraire (relevant du conte) ou un medium philosophique (dans la tradition de Cicéron). La récurrence du motif et l'importance des explications apportées au phénomène, notamment dans les Visions d'Ibraïm, qui en dépit de leur fantaisie se fondent sur d'importantes sources médicales ${ }^{21}$, suggèrent que cet état, et par extension sa transcription-invention littéraire, correspond à un moment où quelque chose pense en nous et, peut-être, s'approcherait d'une forme de vérité - à tout le moins d'une théorie qui vaudrait bien les autres. Cet apparent secret du songe tiphaignien, conçu comme une façon de voir autrement, est alors à mettre en relation avec sa théorie des esprits animaux.

\section{Une explication médicale : esprits animaux et théorie de la sympathie}

Dans les Visions d'Ibraïm, Tiphaigne fonde sa théorie de la différence entre veille et sommeil, et ainsi du rêve, sur une analyse médicale du système nerveux héritée notamment de Macrobe et de Malebranche : le sommeil correspond à une cessation du mouvement qui affecte le «fluide subtil » des sentiments et des pensées qui se trouve dans le cerveau. Ce fluide subtil, c'est celui des esprits animaux, vecteurs des sensations et des idées qu'elles font naître, et qui circule dans des «sillons » ou des «traces ». Le rêve correspond à une cessation partielle de cette circulation, avec une concentration des esprits animaux en certaines zones :

Quand le cours des esprits est arrêté dans toutes les parties du cerveau, le sommeil est aussi profond qu'il puisse l'être. S'il reste encore quelque canton où les esprits circulent, le sommeil est imparfait : on dort, mais on ne dort pas complètement ; on rêve. Les songes procèdent donc de ce que les esprits animaux, fixés partout ailleurs, coulent encore dans certaines traces ${ }^{22}$.

Les traces en question, conformément à la théorie malebranchiste de l'habitude, sont celles qui correspondent aux préoccupations les plus fortes:

Il n'est point de traces plus profondes que celles qui nous représentent les objets dont nous avons coutume de nous occuper; il n'en est point vers lesquelles les esprits animaux aient une pente plus aisée, et qu'ils parcourent plus fréquemment. Elles doivent donc, ou nulle autre, échapper à l'affaissement universel qui survient au cerveau dans le temps du sommeil ${ }^{23}$.

10 Le processus physiologique ainsi décrit - tout à fait recevable au regard de la science de l'époque - constitue une entrée en matière, servant un autre propos : l'analyse des 
rêves, c'est-à-dire des « combinaisons » nouvelles que font les esprits animaux dans les «traces » laissées par les idées de la veille. Difficile de savoir si les exemples que fournit ensuite le texte des Visions sont là pour réellement illustrer le propos médical, ou si cette théorie sert de postulat invitant à faire des contes imaginatifs autour du thème du rêve. Dans un retournement du processus démonstratif, l'exemplum prend en effet son autonomie et devient une fable à plaisir où les esprits animaux déploient le rêve et où le rêve explique le monde. Dans la lignée de Cyrano de Bergerac, Tiphaigne joue de ces renversements qui brouillent les frontières. Le rêve devient supérieur à la veille, parce que les plaisirs (certes, aussi les peines) y sont démultipliés et bien réels puisque procurés par les mêmes esprits animaux que lorsqu'on est éveillé. L'état de veille est considéré par rapport au sommeil, conçu comme état premier : « ce qu'on appelle être éveillé, n'est que dormir un peu moins ${ }^{24} »$; le renversement autorise la continuité. Car les esprits animaux sont ceux qui perçoivent les sensations et les mènent à la fibra patiens, mais aussi ceux qui véhiculent les idées issues de ces mêmes sensations. Le rêve n'est alors pas un processus purement "imaginatif », mais bien physique, fondé sur les sensations et les idées de la veille ou de l'habitude, réactivées, voire renforcées par cette cessation du mouvement. D'où leur prégnance : le rêve est un phénomène avant tout physique. Ainsi, la fiction qui y naît tient du réel sensible et peut bien déceler une vérité cachée au savant éveillé, qui « voit mal ». Plus largement, que le texte se donne explicitement ou non, nous y reviendrons, comme onirique, l'écriture merveilleuse, chez Tiphaigne, à l'instar du songe des humains, repose sur un phénomène de sensibilisation accrue qui permet de sentir ou de percevoir tous les liens invisibles de la nature.

11 Or, dit Ibraïm, « il y a peu de différence (quant aux opérations) entre un homme qui dort profondément et une plante: il végète seulement ${ }^{25}$ ». L'emploi du verbe "végéter ", loin d'être anodin, implique une continuité des règnes, de l'humain au végétal (en passant par l'animal). Déjà, dans Amilec, les germes humains autrement dits "graines d'homme" sont cultivés par les génies dans des "tubules végétaux", suggérant, selon Emmanuelle Sempère, « que tout procède par végétation ${ }^{26}$ et ce, aussi bien dans l'homme et l'animal que dans le végétal. Aussi les "tubules végétaux des animaux" permettent-ils d'imaginer les conditions de possibilité d'une régénération des tissus, voire d'une reproduction généralisée par bouture ${ }^{27} »$. La réflexion du médecin normand se trouve ici fertilisée par les expériences de Leeuwenhoek et par les travaux récents sur le polype. Dans le débat entre mécanistes et vitalistes, il penche sensiblement pour la seconde thèse qui repose sur une continuité des règnes. Dès 1759, il consacre un ouvrage, sérieux cette fois, à l'agriculture, où il développe l'idée d'une sensibilité des plantes équivalente à celle des animaux: "Voyons donc les végétaux comme des êtres sensibles, et regardons ceux d'entre eux qui nous environnent comme nos contemporains et nos compatriotes: la Nature animée de toute part, n'en deviendra que plus intéressante ${ }^{28}$. »Certains développements pourraient être tenus par le docteur Bordeu du Rêve de D'Alembert (et peut-être même par le vrai) :

Un paquet de fibres entre les mains de la nature peut faire le corps d'un homme ou celui d'une plante. C'est toujours la même chose quant au fond, et qu'est-ce que différer par la forme? Qu'importe que ces fibres fassent une partie osseuse ou une partie ligneuse, une main ou une branche, une peau ou une écorce ${ }^{29}$ ?

Cette «fibre " originelle n'est pas explicitement la fibra patiens où convergent, dans l'homme, les esprits animaux, mais le glissement analogique est déjà possible. Dans l' Histoire des Galligènes, les plantes sont ainsi désignées par la périphrase «animaux 
silencieux ${ }^{30}$ ». Cette épithète, qui évoque le dormeur d'Ibraïm, laisse entendre que la frontière entre plante et animal est aussi ténue qu'entre la veille et le sommeil, car, dans le fond : «Est-ce que des individus qui naissent, vivent et meurent, qui croissent et multiplient, qui sont sains et malades, peuvent être autre chose que des animaux ${ }^{31}$ ?» Emmanuelle Sempère a d'ailleurs montré comment l'Histoire des Galligènes reproduisait, au second volume, le débat entre Locke et Leibniz sur la sensibilité animale. Il semblerait en fait que la réponse soit offerte par les plantes merveilleuses cultivées par les Galligènes, que le récit donne à voir. La plante sensitive y a des parentes éloquentes comme "le plaintif ", "l'arbre filtrant» ou encore le "lin aérien », mais surtout cet « arbrisseau d'hauteur d'homme » qui a reçu de la nature le « don de la voix, afin qu'en manifestant ses douleurs, [il] pût attendrir l'homme et l'intéresser à son sort $»^{32}$. À l'instar du travail d'imagination de l'auteur, le labeur de l'agriculteur galligène a permis de rapprocher sensiblement les règnes. Nul besoin de manger la plante pour "l'animaliser ${ }^{33}$ »: plutôt que d'«assimiler », il s'agit, pour Tiphaigne, de rendre similaire.

En revanche, il ne descend pas dans cette chaîne jusqu'au marbre ou à la pierre. Son univers sensible est celui des êtres vivants et, surtout, loin du matérialisme ${ }^{34}$, il repose sur un principe spirituel, puisque ce sont les esprits animaux qui font la sensibilité. Dans L'Amour dévoilé, la "matière sympathique est partout». Or, les esprits animaux, comme la matière sympathique sont des concepts dont la scientificité relative au XVIII siècle est à peu près équivalente à celle de l'atome qui n'aura pas d'existence « légale » avant les travaux de Dalton. Mais cette notion d'« esprit " permet à l'auteur non seulement de créer un univers ultrasensible, mais aussi de lui donner de l'« intelligence » : loin de la physique atomiste - qu'elle rejoint par son souci d'unité -, la nature chez Tiphaigne relève d'une unité et d'une cohérence invisibles aux humains, qu'ils soient naturalistes ou physiciens, mais accessibles aux rêveurs et aux poètes.

\section{Des esprits animaux aux esprits élémentaires : l'au- delà de la sensation}

Qu'ils adoptent explicitement (comme Amilec) ou non la forme du songe, les contes de Tiphaigne en reprennent certains éléments structurels. Le Voyage aux Limbes s'ouvre sur un chapitre intitulé « Ne demandez pas encore comment cela s'est pu faire » qui voit le héros-narrateur emporté " par une force irrésistible [...] à plus d'une demi-lieue au-dessus de la surface de la Terre ${ }^{35}$ »; et c'est seulement à son retour sur Terre, accroché à un des plis de la robe du Génie Azariel, qu'il explique au lecteur qu'en fait il était quasi-mort et que son esprit était parti involontairement pour ce voyage astral ${ }^{36}$, comme en rêve. Les séquences de début et de fin de Giphantie relèvent bien a priori du voyage imaginaire, mais on y retrouve des éléments oniriques: le héros voyage en Guinée, est emporté par un tourbillon, s'assoupit et se réveille dans une région inconnue, celle des esprits élémentaires. Son retour à travers des souterrains et des ruisseaux alterne obscurité et lumières éblouissantes pour le ramener "à six cents stades de Babylone ${ }^{37} »$ (donc de Paris). Cet onirisme caractéristique des contes tiphaigniens est ce qui autorise le glissement métonymique entre la perception "habituelle» du monde et celle qui se fait jour dans ses œuvres; c'est ce flou paradoxalement appuyé par le discours savant - préfaciel ou enchâssé - qui assure la continuité des règnes et la cohérence entre le réel et le merveilleux. Se situant entre 
veille et sommeil, le narrateur voit enfin ce que la lecture des livres savants ne lui permettait pas de concevoir. Si les physiciens des Limbes ne peuvent apercevoir les esprits animaux, le rêveur des contes parvient quant à lui à rencontrer les esprits élémentaires.

Les génies élémentaires d'Amilec expliquent la génération humaine, mais force est de constater, au terme des théories qui s'enchaînent et s'entremêlent (ovisme, emboîtement, dissémination, épigénèse), que le phénomène n'a en fait plus guère besoin des génies que pour recueillir les germes humains, ce que la nature des corps fait pourtant si bien toute seule. La fiction des génies apparait donc comme le processus de germination poétique permettant d'aboutir à cette nouvelle théorie de la génération et donc au savoir. Car c'est bien là ce qui caractérise l'esprit élémentaire chez Tiphaigne : il « incarne » (si l'on peut dire), comme chez Gabalis (auquel fait référence l'auteur ${ }^{38}$ ), une connaissance supérieure, notamment au moyen d'une perception accrue du réel. En 1761, L'Empire des Zaziris sur les humains ou la Zazirocratie propose une théorie merveilleuse et satirique fondée sur des esprits élémentaires invisibles dont l'action consiste aussi à faire agir les hommes :

Avant l'An un, qui commença la généalogie des temps, le Souverain Être créa, depuis lui jusqu'à l'insecte, des multitudes innombrables d'Esprits, aussi diversifiés que nos visages. Il voulut qu'il y eût, de classe en classe, des Génies qui dominassent les uns sur les autres; et que ceux qui vivent unis à la nature des Éléments, se servissent de nous pour leur plaisir et leur utilité, comme nous nous servons des animaux. Ainsi l'homme se joue du singe, et les Zaziris s'amusent de l'homme ${ }^{39}$.

Ces Zaziris ressemblent par bien des traits aux esprits élémentaires qui agissent sur l'esprit humain et font évoluer ses connaissances dans Giphantie, publié l'année précédente. Ils ont le pouvoir de tout savoir et de tout voir ou presque, et à défaut se servent d'artefacts mi-scientifiques, mi-merveilleux, comme cet instrument qui permet d'apprendre ce qui se déroule en n'importe quel endroit de la Terre, simplement en pointant une baguette sur un point d'un globe :

De petits canaux imperceptibles [...] viennent, de chaque point de la superficie de la terre, aboutir à ce globe. Son intérieur est organisé de manière que l'émotion de l'air qui se propage par les tuyaux imperceptibles et s'affaiblit à la longue reprend de l'énergie à l'entrée du globe et redevient sensible ${ }^{40}$.

Les tuyaux de cet étrange appareil évoquent immédiatement au lecteur de Tiphaigne ces tubules, fibres, sillons, traces et canaux qui permettent la circulation des esprits animaux dans les plantes, les animaux et les hommes. Plus qu'une «intuition anticipatrice [...] de la caméra de télévision ${ }^{41} »$, il s'agit d'un prolongement métonymique de la circulation des esprits animaux dans le corps humain à celui du monde - grand animal - que seuls les esprits élémentaires peuvent opérer : ils deviennent le lien invisible de la grande chaîne des êtres, à l'instar des esprits animaux qui relient le corps à l'âme ${ }^{42}$.

17 Sur ce point, nous rejoignons la thèse d'Yves Citton, pour qui l'analogie entre esprits animaux et esprits élémentaires est centrale dans l'œuvre de Tiphaigne de La Roche ${ }^{43}$. Et cette interprétation, fondée d'abord sur La Zazirocratie et les contes mettant en scène les esprits élémentaires, trouve aussi sa justification dans les textes plus sérieux de l'auteur. Il ne s'agit pas seulement d'une exploitation de la richesse polysémique du mot «esprit » au XVIII ${ }^{\mathrm{e}}$ siècle $\mathrm{e}^{44}$, mais bien d'une conception profonde de l'auteur qui la développe à plusieurs reprises. Dans L'Amour dévoilé, la «matière sympathique " relie les corps par les airs : les esprits animaux qui s'exhalent des corps communiquent selon 
un procédé proche de celui des harmonies vibratoires de la musique ${ }^{45}$. Le monde se retrouve ainsi habité d'esprits divers qui relient les corps entre eux et le corps à l'âme grâce à la fibra patiens. C'est un univers spiritualiste et unifié qu'il défend aussi dans l' Essai sur la nature de l'âme où il réfute le matérialisme radical :

Il ne faut pas chercher à comprendre comment un corps peut agir sur un esprit, ni celui-ci sur l'autre. Il faut examiner si un corps peut penser ; car s'il ne le peut pas, il faudra admettre l'union des corps et des esprits et une action mutuelle des uns sur les autres, quoiqu'on ne puisse comprendre ni cette union ni cette action réciproque. (\$ II, VIII) ${ }^{46}$

[...]

Vous auriez tort, avons-nous dit de notre côté, de nier l'existence des purs esprits et leur union à des corps, sous prétexte que vous ne pouvez vous figurer ni ces esprits, ni cette union. Je ne comprends pas cela, donc Dieu ne le peut faire: qui osera raisonner ainsi ? (Conclusion $\S \mathrm{II})^{47}$

Aux esprits animaux qui font sentir, penser et rêver les êtres vivants, correspondent les esprits élémentaires les faisant agir, comme le signale l'étymologie fantaisiste du mot « Zaziri », prétendument emprunté au chinois :

Ces génies ont je ne sais combien de sens que nous n'avons pas; et ceux qui influent sur nos plaisirs, nos malheurs, et même sur notre santé, s'appellent Zaziris, qui en Chinois, veut dire Agents ${ }^{48}$.

De même, aux théories sérieuses qui agitent les savants, correspondent les hypothèses merveilleuses de Tiphaigne, réécritures oniriques parfois plus pertinentes qu'elles n'en ont l'air. Le rêve devient cet agent de communication ${ }^{49}$ qui permet de percevoir le monde autrement, et peut-être un peu comme il va. Car le songe, avons-nous vu, est conçu par le médecin normand comme un phénomène physiologique, fondé sur les sensations (et les idées qui en découlent) de la veille ou de l'habitude, qu'il réactive et transforme pour donner à voir ou à sentir différemment. Le songe serait une machine à transformer les perceptions, mais pas une machine au sens cartésien. L'imagination merveilleuse, ainsi redéfinie, joue le rôle d'une "toile médullaire " (pour reprendre l'expression de La Mettrie ${ }^{50}$ ) où se retissent les fils parcourus par les esprits animaux. Et c'est ce processus qui fonde d'une manière générale l'écriture du merveilleux chez Tiphaigne. La fiction est alors véritablement heuristique : en ce qu'elle permet non pas un savoir nouveau, mais la germination de la pensée permettant d'accueillir un savoir nouveau, fondé sur des perceptions revisitées. À l'instar du songe, elle correspond poétiquement - et peut-être même épistémologiquement - à cette interface entre le monde de la physis et l'âme. Mais tout ceci n'est qu'un jeu, répète l'auteur, un mélange de «sérieux et de badinage, de fables et de vérités [afin d']égayer ces hommes flegmatiques, qui voudraient toujours penser; faire penser de temps en temps ces esprits frivoles, qui voudraient toujours s'égayer ${ }^{51} »$. Et le système développé par Tiphaigne relève lui-même d'une plaisante aporie : les esprits animaux expliquent le phénomène du songe tandis que le songe - ou la vision - nous fait voir les esprits élémentaires et, ainsi, se retrouve à l'origine de l'explication savante. La fiction merveilleuse, comme le réel, tout ne devient alors qu'un spirituel jeu sur les vues de l'esprit humain. 


\section{NOTES}

1. Ph. Vincent, "Sympathie(s) et fiction dans l'œuvre de Charles Tiphaigne ", TrOPICS, $\mathrm{n}^{\circ} 1$, «Fictions et Sciences », G. Armand (dir.), 2013, p. 91-103.

2. Descartes, Les Passions de l'âme [1649], art. X, CEuvres et lettres, Paris, Gallimard, coll. «Bibliothèque de la Pléiade ", 1937, p. 699.

3. Elle se trouve, au $\mathrm{XVIII}^{\mathrm{e}}$ siècle, en concurrence avec les hypothèses électriques qui vont progressivement gagner du terrain. Voir F. Panese, "Les "esprits animaux" au défi de l'expérience. Enquête sur un objet de connaissance en voie de disparition au $18^{\mathrm{e}}$ siècle ", dans I. Laboulais et M. Guédron (dir.), Écrire les sciences, Éditions de l'Université de Bruxelles, coll. «Études sur le XVIII ${ }^{\mathrm{e}}$ siècle », 2015, p. 15-30.

4. Cette notion, comme le montre l'évolution sémantique du mot (qui sert aussi bien, de nos jours, en médecine que dans la langue courante), est au XVIII siècle déjà fortement polysémique, ce qui autorise en quelque sorte non seulement qu'elle intervienne dans différents domaines du savoir, mais aussi et surtout qu'elle entraîne un certain flou conceptuel, dû aux nombreux glissements de sens que lui font subir les auteurs. Pour plus d'information sur la question, voir M. A. Bernier, "Les métamorphoses de la sympathie au siècle des Lumières ", dans M. A. Bernier et D. Dawson (dir.), Les Lettres sur la sympathie (1798) de Sophie de Grouchy: philosophie morale et réforme sociale, Oxford, Voltaire Foundation, 2010. Voir également T. Belleguic, E.van der Schueren et $\mathrm{S}$. Vervacke (dir.), Les discours de la sympathie. Enquête sur une notion de l'âge classique à la modernité, Québec, PUL, 2007 [rééd. Paris, Hermann, 2014].

5. Fl. Boulerie, « La philosophie admet-elle la fantaisie du style? », Br. Curatolo et J. Poirier (éds), Le style des philosophes, Besançon, PUFC, 2007, p. 133-142. Elle va jusqu'à voir un jeu d'influence entre les deux auteurs, en dépit de la différence de pensées : dans la continuité de la tradition critique (J.Marx, notamment), elle établit que Tiphaigne est certainement influencé par le Diderot des Pensées philosophiques, mais elle pose aussi l'hypothèse que les œuvres de Tiphaigne pourraient avoir influencé l'écriture du Rêve de D'Alembert.

6. Correspondance Littéraire, Philosophique et Critique Par Grimm, Diderot Raynal, Meister, Etc., M. Tourneux (éd.), Paris, Garnier Frères, 1878, t. IV, p. 127.

7. Cet ouvrage, Les Bigarrures philosophiques (1759), est, conformément à ce qu'annonce son titre, un ouvrage assez composite qui s'ouvre sur un traité du sommeil prétendument traduit de l'arabe, suivi d'un essai sur la nature de l'âme qui opère une réfutation de Locke, mais s'interrompt à la fin du premier volume pour ne reprendre qu'à la fin du second, juste après le Voyage aux Limbes. Pour une présentation plus complète de l'ouvrage, nous nous permettons de renvoyer à notre édition: Tiphaigne de La Roche, Les Bigarrures philosophiques, G. Armand et E. Sempère (éd.), Euvres complètes, J. Marx (dir.), Paris, Classiques Garnier, coll. « Lire le Dixhuitième siècle ", sous presse.

8. Voir notamment G. Armand, "Idée d'une République Philosophique: l'impossible utopie solaire de Cyrano », Expressions, $n^{\circ}$ 25, juin 2005, p. 63-80.

9. Voir notamment G. Armand, «La science du Père Daniel et les fictions de Descartes », dans J. Ducos (dir.), Les sciences et le livre. Formes des écrits scientifiques des débuts de l'imprimé à l'époque moderne, Paris, Hermann, 2017, p. 299-315.

10. Tiphaigne de La Roche, Bigarrures philosophiques, Amsterdam, Chez Arkstée et Merkus, 1759, t. 2, p. 33-34 (nous soulignons).

11. Ibid., p. 163.

12. Tiphaigne de La Roche, Giphantie [1760], éd. Fr. Lacassin, Voyages aux pays de nulle part, Paris, Robert Laffont, 1990, p. 1025. 
13. Sur les différentes éditions d'Amilec, voir l'introduction de Philippe Vincent à l'édition de ce texte, Amilec ou la graine d'hommes, Mont-Saint-Aignan, PURH, coll. « Lumières normandes ", 2012, notamment les pages 71-84.

14. Voir à ce propos G. Armand, "Tiphaigne de la Roche ou la vocation fantaisiste de la science », dans Y. Citton, M. Dubacq et Ph. Vincent (éds), Imagination scientifique et littérature merveilleuse. Charles Tiphaigne de La Roche, Pessac, PUB, coll. "Mirabilia», 2014, p. 193-209; Ph. Vincent, «Charles Tiphaigne et la génération dans Amilec, ou la graine d'hommes (1754) », Féeries, n 6, 2009, p. 107-115 (disponible sur http://journals.openedition.org/feeries/703) ; E. Sempère, « Tiphaigne de la Roche entre science et merveille ", Féeries, n6, 2009, p. 117-130 (disponible en ligne : http:// journals.openedition.org/feeries/706).

15. Tiphaigne de La Roche, Amilec ou la graine d'hommes, éd. citée, p. 95.

16. Tiphaigne de La Roche, Bigarrures philosophiques, ouvr. cité, t. 1, p. 25.

17. Ibid., p. 5.

18. Tiphaigne de La Roche, Amilec ou la graine d'hommes, éd. citée, p. 95 : «La barrière était ouverte, le champ était vaste ; j'aurais, sans doute, porté fort loin ces réflexions. Mais au milieu de ma boutade [...] je m'endormis ; je fis plus, je rêvai. »

19. Tiphaigne de La Roche, Amilec ou la graine d'hommes, éd. citée, p. 93 : «Autrefois, je lisais, je réfléchissais, je combinais, je mettais mon esprit à une torture qui en fatiguait les ressorts, et je n'apprenais rien. Aujourd'hui, je me tranquillise, je dors, je rêve et je deviens savant. Que ne l'aije su plutôt que pour faire des systèmes et des découvertes, il ne s'agissait que de rêver philosophiquement. »

20. On retrouve cette idée, par exemple au tout début de La Zazirocratie: "Ce Système, tout ridicule qu'il paroît au premier abord, satisfait à toutes les difficultés ; \& d'ailleurs si l'on passe à Descartes sa matière subtile, à Newton son attraction, à Leibnitz [sic] ses monades, il me semble qu'on pourra bien me permettre une hypothèse, avec laquelle j'explique tous les hasards, toutes les contradictions de la vie, \& je fais rouler, de la meilleure grace du monde, la terre \& les cieux. » L'Empire des Zaziris sur les humains, ou la Zazirocratie, Pékin, chez Dsmgtlfpqxz, 1761, p. 2-3.

21. On pense notamment à Boerhaave (voir à ce sujet, notre introduction, éd. Classiques Garnier, à paraître).

22. Tiphaigne de La Roche, Bigarrures philosophiques, ouvr. cité, t. 1, chap. V «Demi-sommeil », p. 30-31.

23. Ibid., chap. IX « Être heureux en songes, c'est l'être plus qu'on ne pense », p. 54-55.

24. Ibid., chap. IV, p. 25.

25. Tiphaigne de La Roche, Les Visions d'Ibraïm, ouverture du chap. IV "Avis aux beaux esprits » (Bigarrures philosophiques, ouvr. cité, t. 2, p. 19).

26. Le Dictionnaire de l'Académie définit "végéter» par «croître, pousser par un principe intérieur » (1694) [note d'E. Sempère].

27. E. Sempère, "Savoirs scientifiques et imagination littéraire: les plantes sensibles de Tiphaigne de la Roche ", dans I. Laboulais et M. Guédron (dir.), ouvr. cité, p. 145. Sur cette question de la continuité des règnes et des jeux qu'elle entraîne dans l'écriture de Tiphaigne, voir aussi R. Le Menthéour, «De la moisson humaine : botanique et perfectibilité dans les Lumières médicales ", TrOPICS, $n^{\circ}$ 1, « Fictions et Sciences », G. Armand (dir.), nov. 2013, revue en ligne.

28. Tiphaigne de La Roche, Questions relatives à l'agriculture et la nature des plantes, Paris, Neaulme, 1759 , p. 89.

29. Ibid., p. 102-103.

30. Tiphaigne de La Roche, Histoire des Galligènes ou mémoires de Duncan, 1765, Amsterdam et Paris, Vve Durand, 1765, vol. 2, p. 50.

31. Ibid., p. 51.

32. Ibid., p. 54 . 
33. Diderot, Entretien entre Diderot et D'Alembert, Le rêve de D'Alembert, éd. C. Duflo, Paris, Flammarion, coll. «GF », 2002, p. 57 : «Oui, car en mangeant, que faites-vous ? Vous levez les obstacles qui s'opposaient à la sensibilité active de l'aliment ; vous l'assimilez avec vous-même ; vous en faites de la chair ; vous l'animalisez, vous le rendez sensible [...]. »

34. Rappelons que Tiphaigne est bien antimatérialiste, comme le montrent son Essai sur la nature de l'âme, mais aussi nombre d'allusions dans ses contes satiriques et même le principe de ses fictions : ainsi, les «graines » d'Amilec contiennent des «âmes »; la « grotte de Thotis » (Visions d'Ibraïm, chap.XIV-XVI, Bigarrures philosophiques, ouvr. cité, t.1, p. 89 et suiv.) renferme les « âmes de mille et un sages » privés jusqu'alors de lumière.

35. Tiphaigne de La Roche, Voyage aux Limbes, Bigarrures philosophiques, ouvr. cité, t. 2, p. 1-2.

36. Cette idée de voyage astral rapproche encore plus ce récit du Voyage du Monde de Descartes du Père Daniel.

37. Tiphaigne de La Roche, Giphantie, ouvr. cité, p. 1085.

38. Voir, par exemple, dans La Zazirocratie : «On verra que notre opinion diffère entièrement des rêveries du Comte de Gabalis, et que les Génies que nous admettons, étant des substances purement Spirituelles, aussi bien que nos âmes, ne nous font du bien ou de mal qu'à raison des lois générales, qui en ont ainsi ordonné.» (Tiphaigne de La Roche, L'Empire des Zaziris sur les humains, ou la Zazirocratie, ouvr. cité, p. 6)

39. Ibid., p. 1-2.

40. Tiphaigne de La Roche, Giphantie, ouvr. cité, p. 1030.

41. J. Marx, Tiphaigne de la Roche : modèles de l'imaginaire au XVIII siècle, Éditions de l'Université de Bruxelles, 1981, p. 65.

42. Sur ce point, voir notre article : «Des esprits animaux aux esprits élémentaires : physiologie et poétique chez Tiphaigne de La Roche ", dans M. Louis-Courvoisier et S. Kleiman-Lafon (dir.), Les Esprits animaux, Epistémocritique, Revue d'études et de recherches sur les savoirs et la littérature, revue en ligne, printemps 2018.

43. Voir Y.Citton, "Spirits accross the Channel. The Staging of Collective Mental Forces in Gabalistic Novels from Margaret Cavendish to Charles Tiphaigne de la Roche ", Comparatio. Zeitschrift für Vergleichende Literaturwissenschaft, $\mathrm{n}^{\circ}$ 1-2 (2009), Heidelberg Universitätsverlag, p. 291-319; ainsi que, du même auteur, "La science illuministe du merveilleux. Entre roman véritable et roman de magie ", Féeries, n 6, 2009, « Le conte, les savoirs », p. 21-46.

44. Voltaire, art. " Esprit », Encyclopédie de Diderot et d'Alembert : « Il ne faut pas oublier de dire ici en combien de sens différents le mot d'esprit s'emploie; ce n'est point un défaut de la langue, c'est au contraire un avantage d'avoir choisi ainsi des racines qui se ramifient en plusieurs branches. »

45. Ph. Vincent, «Sympathie(s) et fiction dans l'œuvre de Charles Tiphaigne », art. cité, p. 96 : "Tiphaigne utilise les unissons pour expliquer le fonctionnement du système nerveux et pour cela, il utilise la métaphore des instruments à corde. [...] Si la métaphore est filée jusqu'à ses dernières conséquences, la vision du système nerveux développée par Tiphaigne porterait en elle-même la nécessité de l'existence de l'âme. »

46. Tiphaigne de La Roche, Bigarrures philosophiques, ouvr. cité, t. 2, p. 259.

47. Ibid., p. 288.

48. Tiphaigne de La Roche, La Zazirocratie, ouvr. cité, p. 6-7.

49. Nous empruntons l'idée à Yves Citton: "We now understand more precisely in which manner the immaterial spirits can be called "agents": theirs is an agency of communication. Just like the "animal spirits" within the human body are defined by Voltaire, in the Encyclopaedia, as "what has never been seen and what gives movement and life" to the animated body, similarly the elementary spirits represent the influencing factors which communicate movements within human societies. » (art. cité, p. 316).

50. "Je me sers toujours du mot imaginer, parce que je crois que tout s'imagine, et que toutes les parties de l'âme peuvent être justement réduites à la seule imagination, qui les forme toutes ; et 
qu'ainsi le jugement, le raisonnement, la mémoire ne sont que des parties de l'âme nullement absolues, mais de véritables modifications de cette espèce de toile médullaire, sur laquelle les objets peints dans l'œil, sont renvoyés, comme d'une lanterne magique. » La Mettrie, L'Homme machine [1747], Paris, Pauvert, 1966, p. 85.

51. Tiphaigne de La Roche, Bigarrures philosophiques, ouvr. cité, t. 1, p. vi.

\section{RÉSUMÉS}

Conformément à la pensée des Lumières, Tiphaigne de La Roche se méfie des sens, qu'il sait insuffisants pour accéder à la vérité. Mais d'influence empiriste, il ne voit d'autre voie d'accès au savoir. Aussi est-ce paradoxalement l'imagination qui vient au secours de l'homme, notamment à travers le rêve. Plus qu'un simple motif littéraire, le songe devient le lieu où les esprits animaux, porteurs des sensations dans le corps humain (selon les théories médicales de l'époque), vont se libérer et explorer des voies jusqu'alors inconnues. Dans ses fictions merveilleuses, l'imagination agit comme une «toile médullaire " permettant à un savoir de prendre forme, un savoir qui vaut bien les autres systèmes élaborés par les philosophes et les scientifiques - chez lesquels Tiphaigne puise d'ailleurs une partie de son inspiration.

In the same way as many Enlightenments philosophers, Tiphaigne de La Roche knows that humans' perceptions are too weak to gain access to the Truth. However, he is influenced by the Empirist movement and he does not see any other way to knowledge. So, to him, Imagination becomes a solution: dreams are not a mere literary topic in Tiphaigne's fantasies, they become the place where Animal spirits (in accordance with medical theories in the 18th century), get free and explore unknown paths and places to elaborate new theories. In his marvelous fiction, Imagination is what authorizes a new knowledge to be born, a knowledge that can be compared, paradoxically, to the other forms of philosophical and scientific knowledge of the time-that inspired him his delirious theories.

\section{INDEX}

Mots-clés : imagination, merveilleux, esprits animaux, esprits élémentaires, songe, Tiphaigne de La Roche

Keywords : imagination, marvelous, animal spirits, elementary spirits, dream, Tiphaigne de La Roche

\section{AUTEUR}

\section{GUILHEM ARMAND}

Université de La Réunion 